\title{
Atypical teratoid/rhabdoid tumors of the central nervous system: management and outcomes
}

\author{
Michael L. Chen, B.A., J. Gordon McComb, M.D., And Mark D. Krieger, M.D. \\ Division of Neurosurgery, Childrens Hospital Los Angeles; and Department of Neurological Surgery, \\ University of Southern California Keck School of Medicine, Los Angeles, California
}

\begin{abstract}
Object. Atypical teratoid/rhabdoid tumors (ATRTs) represent a relatively newly categorized neoplastic entity. They commonly present in childhood, and have a rapidly progressive clinical course with a survival time of less than 1 year. Treatment regimens have been nonuniform. In this retrospective review of patients with ATRTs who were treated at the authors' institution according to a uniform protocol, the goal was to assess the efficacy of the treatment and its outcome.

Methods. Over a 7-year period, ATRT was diagnosed in 11 patients (six boys and five girls). The median age of the patients was 61 months, and their ages ranged from 3 months to 17 years. Signs and symptoms began, on average, a little more than 1 month before diagnosis and included the following: headache (36\%), nausea and vomiting (46\%), lethargy $(18 \%)$, seizures $(27 \%)$, cranial nerve findings $(46 \%)$, ataxia $(18 \%)$, long tract findings $(18 \%)$, and hydrocephalus (46\%). Tumor location was cortical in four patients, in the pineal region in four, in the posterior fossa in two, and spinal in one. In one patient disseminated disease was revealed on the initial imaging study; seven patients had disseminated tumor subsequently. Treatment consisted of chemotherapy in 11 patients, chemotherapy and local radiation in five, and chemotherapy and craniospinal radiation in three. Six patients are alive, three have died, and two were lost to follow-up review. The mean time to death was 24 months, and ranged from 2 to 67 months. Among the surviving patients the mean duration of follow up is 18.5 months and ranges from 2 to 37 months. The median time to progression was 3.5 months.

Conclusions. Atypical teratoid/rhabdoid tumors are malignant lesions with rapid progression. Further study is necessary to determine the efficacy of therapy.
\end{abstract}

\section{KEY WORDS • atypical teratoid/rhabdoid tumor • primitive neuroectodermal tumor • medulloblastoma}

Atypical teratoid/rhabdoid tumors are highly malignant CNS neoplasms that arise in children; usually the patients are younger than 2 years of age. These lesions are immunologically distinct from and are resistant to standard therapy for PNETs. ${ }^{2,7,9,11}$ The survival of patients with an ATRT is 6 months on various treatment regimens, with some longer survival times reported in the literature as more intensive therapy is being used..$^{6,8,10,12}$

Although it is becoming clear that ATRTs are distinct entities with specific morphological and immunological characteristics different from those of other CNS neoplasms and malignant rhabdoid tumors, the clinical management of this rapidly progressing disease remains controversial. In this series we summarize the treatment of 11 patients with ATRT at our institution over the last 7 years.

Abbreviations used in this paper: ATRT $=$ atypical teratoid/rhabdoid tumor; CNS = central nervous system; COG = Children's Oncology Group; EFS = event-free survival; MR = magnetic resonance; OS = overall survival; PFS = progression-free survival; $\mathrm{PNET}=$ primitive neuroectodermal tumor.

\section{CLINICAL MATERIAL AND METHODS}

With Internal Review Board approval, a retrospective review of patient records from 1996 to 2003, inclusively, was conducted at the Childrens Hospital Los Angeles, and 11 cases of surgically treated ATRTs were identified. The patient records, which included operative reports, pathology reports, radiological studies, and clinical follow-up visits, were reviewed in their entirety. All patients with this pathological entity who were treated at our institution were included in this study, regardless of associated conditions.

All patients were treated at the inpatient service of the Childrens Hospital Los Angeles. Preoperative imaging studies included an MR image of the entire neuraxis, with Gd-enhanced sequences obtained in all patients.

\section{Patient Population}

Eleven children were treated for ATRT at the Childrens Hospital Los Angeles during the study period. Six (55\%) were boys and five $(45 \%)$ were girls. Their ages ranged 
M. L. Chen, J. G. McComb, and M. D. Krieger

from 3 months to 17 years, with a median age of 3 years. The follow-up period ranged from 2 to 67 months, with a median follow-up duration of 14 months.

Each patient underwent a craniectomy and resection of the tumor, followed by adjunctive radiation and/or chemotherapy. Follow-up MR imaging studies were scheduled at 3-month intervals for 2 years, then at 6-month intervals for 2 years, and were obtained yearly thereafter, or as determined based on clinical symptomatology.

The study variables were OS and PFS. The OS measured the time from the initial surgery to the date of death or the end of the study period, whereas the PFS measured the time from the initial surgery to progression of the tumor (recurrence at the site of origin and/or metastasis) or the end of the study period.

\section{RESULTS}

Ten of the 11 patients presented with neurological signs and/or symptoms, which included the following: five $(45.5 \%)$ with nausea and vomiting, five $(45.5 \%)$ with cranial nerve deficits, four $(36.4 \%)$ with headache, five $(45.5 \%)$ with hydrocephalus, three $(27.3 \%)$ with tonic-clonic seizures, three $(27.3 \%)$ with ataxia, two $(18.2 \%)$ with lethargy, and two (18.2\%) with limb pain. Many patients presented with multiple signs and/or symptoms. One patient was asymptomatic, but a primary tumor was discovered in the third ventricle on follow-up MR imaging for treatment of hydrocephalus. The mean duration of symptoms before admission was 33 days, and ranged from 5 days to 4 months.

Nine of 11 patients had a single cerebral lesion (Fig. 1). Eight of these patients had supratentorial mass lesions and one had an infratentorial mass lesion. One patient presented with mid-back pain due to an intradural spinal cord tumor, and another presented with cranial nerve palsies from a chiasmatic mass lesion and a second cerebellar lesion ${ }^{4}$ (Table 1).

\section{Effect of Treatment on Outcome}

All patients underwent resection of the mass lesion. A
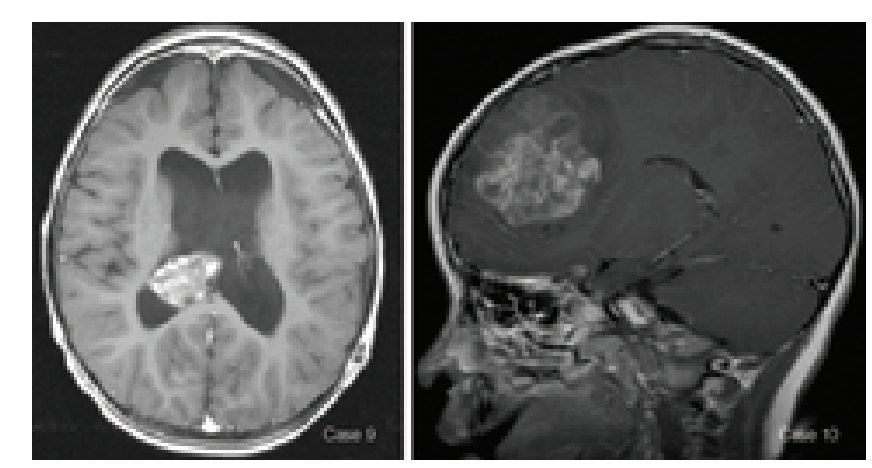

Fig. 1. reoperative MR images obtained in two patients revealing enhancing lesions in the lateral ventricles (Case 9) and frontal lobe (Case 10).

gross-total resection of the tumor was achieved in nine patients, whereas partial resection was achieved in the remaining two. One of them had notable invasion of tumor in the rootlets of the spinal cord, and a total resection was not possible in the other due to the proximity of the tumor to the middle cerebral artery.

All patients underwent multiagent chemotherapy postoperatively (Table 1). Six patients were treated according to the COG 99703 regimen, with three cycles of induction and three of consolidation chemotherapy, which was modified in five patients to include radiotherapy. The patient in Case 1 was treated according to the COG 99701 regimen, which called for simultaneous radiotherapy during the induction phase, followed by maintenance chemotherapy. The patients in Cases 2 and 6 were treated according to the COG 99702 regimen, which called for 6 weeks of craniospinal radiation after induction and stem cell harvest, followed by consolidation with three cycles of chemotherapy. The protocols were similar in the use of cisplatin, vincristine, cytoxan, carboplatin, thiotepa, and etoposide. The patient in Case 5 was treated with carboplatin, adriamycin, cisplatin, and doxorubicin. The patient in Case 11 received

TABLE 1

Clinical characteristics in 11 patients with ATRT*

\begin{tabular}{|c|c|c|c|c|c|c|c|c|}
\hline $\begin{array}{l}\text { Case } \\
\text { No. }\end{array}$ & $\begin{array}{l}\text { Age (yrs), } \\
\text { Sex }\end{array}$ & Lesion Location & $\begin{array}{l}\text { Metastasis } \\
(\mathrm{mos})\end{array}$ & $\begin{array}{l}\text { Time to Pro- } \\
\text { gression (mos) }\end{array}$ & $\begin{array}{l}\text { Extent } \\
\text { of Op }\end{array}$ & $\begin{array}{c}\text { Chemo } \\
\text { (COG No.) }\end{array}$ & Radiation & $\begin{array}{l}\text { Length of FU } \\
(\mathrm{mos}) / \text { Status }\end{array}$ \\
\hline 1 & $1, \mathrm{M}$ & pineal & postop & NA & GTR & 99701 & yes & 24/alive \\
\hline 2 & $15, \mathrm{M}$ & spinal cord & none & NA & STR & 99702 & yes/CS & 34/alive \\
\hline 3 & $5, \mathrm{M}$ & frontal & none & NA & GTR & 99703 & yes/CS & 12/alive \\
\hline 4 & $6, \mathrm{M}$ & It frontotemporal & 24 & 24 & GTR & 99703 & yes/CS & 67/dead \\
\hline 5 & $17, \mathrm{~F}$ & posterior fossa & none & 1 & GTR & 99703 & no & 2/LTF \\
\hline 6 & $0.5, \mathrm{~F}$ & lt frontotemporal & none & 5 & STR & 99702 & yes & 9/LTF \\
\hline 7 & $0.5, \mathrm{M}$ & pineal & none & 11 & GTR & $\dagger$ & no & $12 /$ dead \\
\hline 8 & $2, \mathrm{~F}$ & 3rd ventricle & none & NA & GTR & 99703 & yes & 37/alive \\
\hline 9 & $2, \mathrm{M}$ & 3rd ventricle & 12 & 6 & GTR & 99703 & yes & 14/dead \\
\hline 10 & $7, F$ & bifrontal & none & NA & GTR & 99703 & yes & 27/alive \\
\hline 11 & $0.25, \mathrm{~F}$ & $\begin{array}{l}\text { cerebellar \& } \\
\quad \text { suprasellar }\end{array}$ & none & 2 & GTR & $\ddagger$ & no & 3/dead \\
\hline
\end{tabular}

* Chemo = chemotherapy; $\mathrm{CS}=$ craniospinal radiation; FU = follow up; GTR = gross-total resection; LTF = lost to follow up; $\mathrm{NA}=$ not applicable; STR = subtotal resection .

$\uparrow$ Carboplatin, adriamycin, cisplatin, and doxorubicin.

$\$$ Cisplatin, etoposide, vincristine, cytoxan, and methotrexate, followed by ifosphamide, carboplatin, and etoposide. 
a modified Head Start protocol (cisplatin, etoposide, vincristine, cytoxan, and high-dose methotrexate), followed ifosphamide, carboplatin, and etoposide.

The median duration of treatment was 6 months. Eight patients received cranial and three received craniospinal radiation. Three patients received no radiation in the postoperative period. Of these, the patient in Case 5 was lost to follow up at 2 months, and there was no documentation regarding initiation of radiotherapy; parents of the patient in Case 7 elected to forgo radiation; and the one in Case 11 died of disease before radiation therapy was initiated. The three who received craniospinal radiation had a mean total dose of $52 \mathrm{~Gy}$, whereas those who received local radiation had 44 Gy. The lower mean dose for local radiation therapy was due to the fact that the patient in Case 9 traveled abroad for radiation therapy and only received $27 \mathrm{~Gy}$ in the tumor bed.

At the end of the study period, five patients $(45.5 \%)$ were alive and free of disease, one $(9.1 \%)$ was alive with progression, three $(27.3 \%)$ had died, and two $(18.2 \%)$ were lost to follow up. The median follow-up duration was 24 months, ranging from 2 to 67 months. The tumor progressed postoperatively in five patients $(45.5 \%)$. Time to progression ranged from 1 to 11 months, with a median of 6 months. Metastatic disease developed in three patients. The median time to metastasis was 12 months, ranging from immediately postoperatively to 24 months. One patient with evidence of tumor seeding of the spinal cord on postoperative MR imaging was alive and disease free at the end of the study period. In contrast, the remaining two patients, in whom spinal metastases developed at 12 and 24 months postoperatively, had died at 14 and 67 months, respectively. Metastatic lesions were again resected and chemotherapy was administered.

The mean age at diagnosis of the five patients who were alive and disease free at the end of the study period was 6 years. Treatment included chemotherapy as outlined in Table 1, gross-total resection in four of the five patients and subtotal resection in the other, and craniospinal radiation in two, whereas the remaining patients received local radiation to the tumor bed.

\section{Extent of Radiation and Patient Outcome}

Figure 2 shows the patient outcome divided by the extent of radiation therapy. Eight patients received some form of radiation postoperatively. The three who received craniospinal radiation had the longest mean PFS and OS times (23.3 and 37.7 months, respectively). The median age at diagnosis for this group was 6 years. The remaining five patients, whose median age was 2 years, were treated with local cranial radiation to the tumor bed. The mean PFS and OS times for this group were 19.8 and 22.2 months, respectively. Finally, three patients did not receive radiation therapy. The median age for this group was 7 months, and the mean PFS and OS times were 4.6 and 5.6 months, respectively. Statistical analysis according to the Fisher exact test showed no significant difference among groups based on the extent of radiation and survival at an alpha value of 5\% for PFS, whereas the same analysis of OS showed a significant difference in survival between patients who received craniospinal radiation postoperatively and those who did not (Fig. 2B).

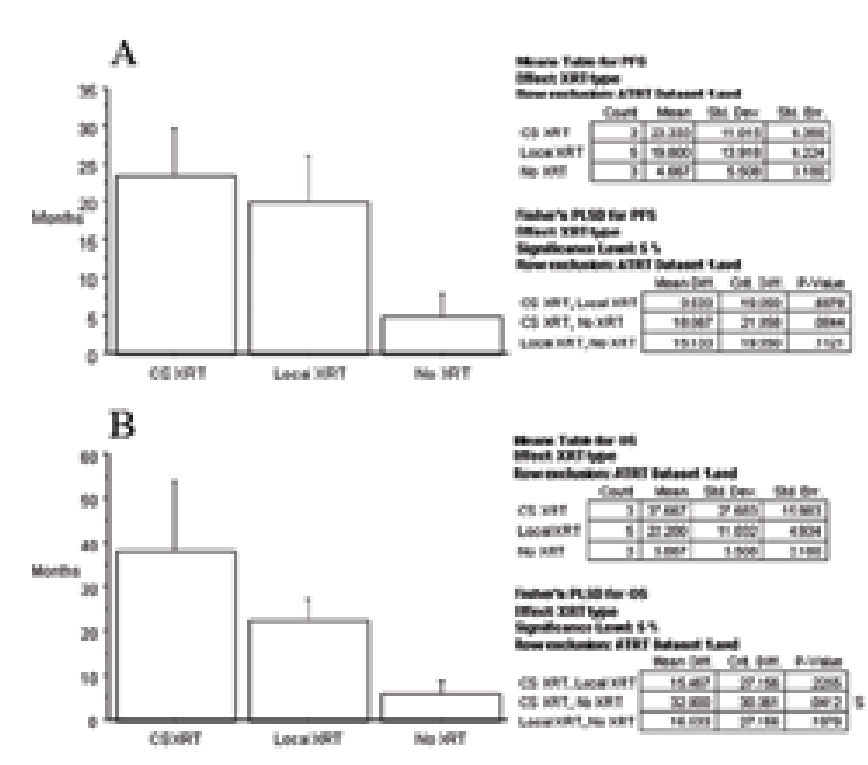

Fig. 2. Bar graphs showing the extent of radiation and survival outcomes. A: Graph showing the PFS rate in patients who received craniospinal (CS) radiation therapy. B: Graph showing the OS rate in patients who received craniospinal radiation therapy. Data are displayed as the mean, standard deviation, and standard error. Crit. Diff. = critical difference; PLSD = protected least significant difference; Std. Dev. = standard deviation; Std. Err. = standard error of the mean; XRT $=$ radiation therapy.

\section{DISCUSSION}

First described in 1987, ATRT was subsequently defined in 1996 as a malignant CNS tumor of infancy containing rhabdoid tumor cells next to areas of PNET. ${ }^{11}$ Typical nests of rhabdoid cells contain large polygonal cells with eosinophilic cytoplasm and eccentric round nuclei with prominent nucleoli (Fig. 3). Histologically, ATRTs resemble PNETs and have frequently been misdiagnosed as such in the past. ${ }^{3,7}$ Furthermore, advances in immunohistochemistry in recent years have allowed pathologists to distinguish ATRTs, making diagnosis possible (Fig. 4)., ${ }^{2,9}$ What remains uncertain is the clinical management of the disease in patients with ATRT.

Rorke, et al., ${ }^{11}$ report that despite providing adjuvant chemotherapy and radiotherapy postoperatively, the median period of survival for 52 patients in their series was 6 months. The main cause of death was progression of the disease and leptomeningeal spread. The patient with the longest survival time had received craniospinal therapy, lived for 5.5 years postradiation, and died of metastatic recurrence. Burger, et al., ${ }^{3}$ summarized 54 patients diagnosed with ATRT in 1998 and found that the mean survival duration was only 11 months when patients were treated with multiagent chemotherapy. Moreover, the ability to distinguish this tumor from PNET has a significant impact on clinical management, because studies have shown that ATRT does not respond to treatment in a manner similar to PNET.

Some hope has come from the use of intensified therapy for ATRT. Olson, et al. ${ }^{8}$ described the use of an intensive regimen (cisplatin, doxorubicin, and vincristine + actinomycin + cyclophosphamide [VAC]/VAC + cisplatin and 


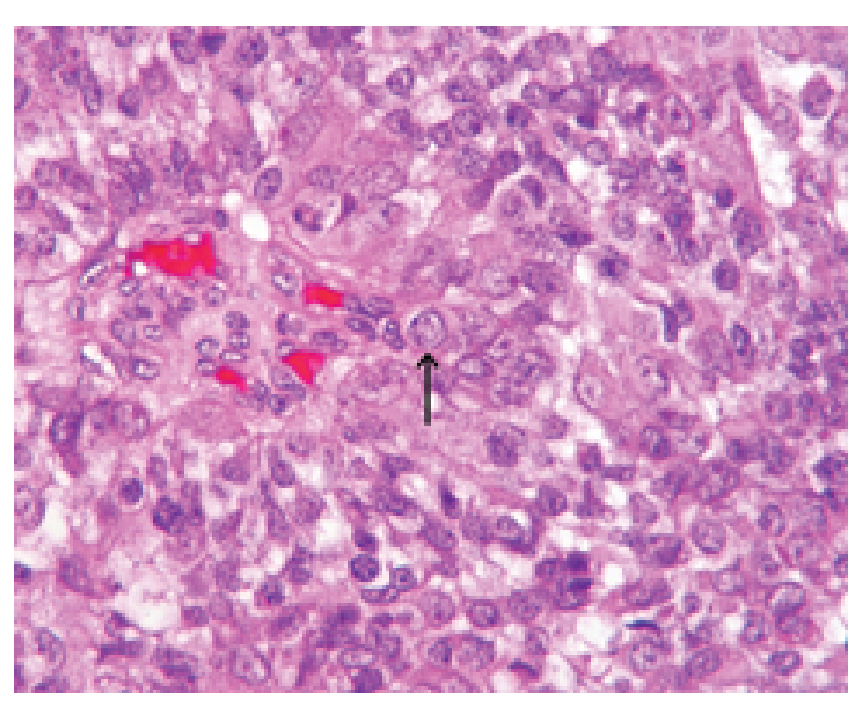

Fig. 3. Case 11. Photomicrograph showing a typical nest of rhabdoid cells with large polygonal cells, eosinophilic cytoplasm, and eccentric round nuclei with prominent nucleoli resected from the cerebellum. The arrow indicates a characteristic rhabdoid cell among the field of PNET cells. $\mathrm{H} \& \mathrm{E}$, original magnification $\times 100$. vincristine + adriamycin + cyclophosphamide [VAdrC]) followed by a course of triple intrathecal therapy to prevent CSF seeding, and then by radiotherapy in three patients with ATRT. The patients were alive and disease free at the end of the study. In 1998, Hilden, et al., ${ }^{6}$ reported on four patients who had received a similar intensified chemotherapy regimen (cisplatin, etoposide, vincristine, ifosfamide, and doxorubicin), radiotherapy, and triple intrathecal therapy. Bone marrow transplantation was attempted in patients with progressive disease. Two of the children died, one at 9 months and the other at 20 months after diagnosis, from disease progression without bone marrow transplantation. Another child was disease free 46 months after diagnosis.

Radiation therapy has been used in patients with ATRT as an adjuvant to resection. In 1998, Weiss, et al., ${ }^{12}$ reported on three cases of ATRT and reviewed the literature. They found that patients who received radiation doses higher than 30 Gy survived a mean of 16 months, and those who received lower doses or no radiotherapy survived a mean of 6 months. One report exists of the use of gamma knife surgery performed in patients with ATRT that resulted in local control of the postoperative lesion, only to see it progress to diffuse leptomeningeal spread of the disease. ${ }^{1}$ The authors of this study concluded that gamma knife surgery may be used in select cases in which the systemic effects of radiation would be detrimental, for example in very young patients.

In 2004, Hilden, et al., 5 reported on 42 patients included in a national ATRT registry. The results confirmed that older children diagnosed with this disease have a better prognosis. The investigators showed an EFS duration of 7.75 months in children younger than 3 years of age, whereas children older than 36 months had a median EFS of 16 months. In this group of patients, the majority under-

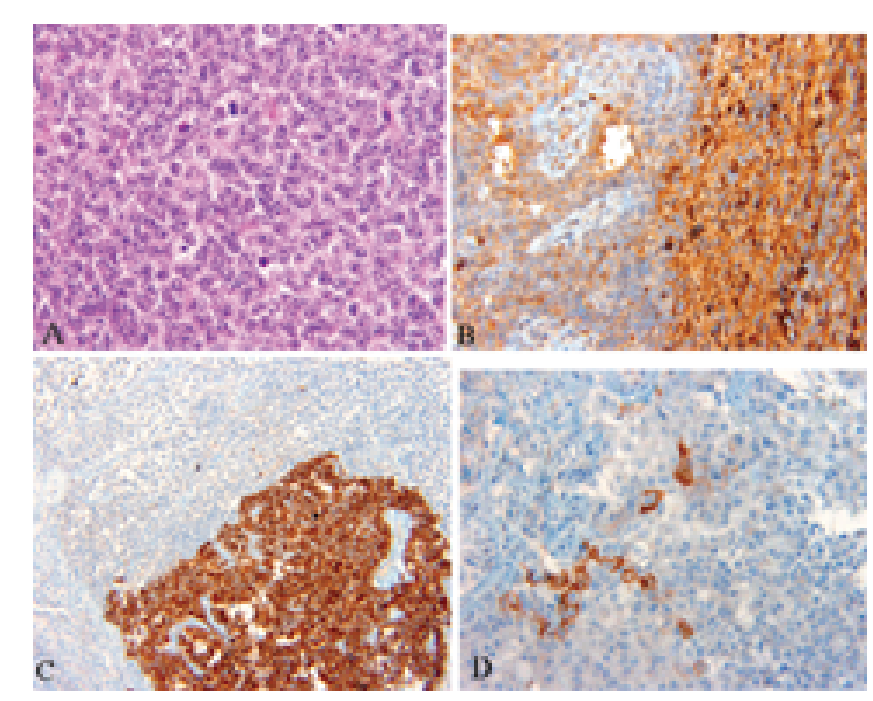

Fig. 4. Case 11. Sections of a cerebellar tumor stained for histological investigation. A: Section of tumor tissue showing characteristic rhabdoid cells. B-D: Sections from the same tumor showing cytoplasmic staining. H \& E (A), glial fibrillary acidic protein (B), keratin (C), and smooth muscle actin (D). Original magnification $\times 100$. went resection and chemotherapy as their primary treatment. Those who also received intrathecal chemotherapy (38\%) had an EFS duration of 16.5 months and an OS time of 23 months. Those with high-dose chemotherapy and stem cell rescue had an EFS duration of 10 months and an OS time of 21.5 months. Finally, $31 \%$ of this group received radiation therapy. The EFS duration in the patients treated with radiation was 48 months, and the OS time was 48 months. Of note, the subgroup that also received radiation therapy had a mean age of 47 months. The authors concluded that radiotherapy remains controversial in the treatment of ATRT but maintain that it may contribute to disease control.

Craniospinal radiation has frequently been used in cases of PNET/medulloblastoma because of its ability to control meningeal dissemination. Our findings indicate a trend in the extent of radiation in association with overall and disease-free survival among patients with ATRT (Fig. 2); however, a more detailed analysis of the relationship needs to be explored. At present, there have been no reports on the effectiveness of craniospinal and localized radiation therapy in the management of ATRT.

\section{CONCLUSIONS}

In this paper we present a case series of patients with ATRT who were treated in a uniform fashion at one institution. We found ATRT to be a malignant embryonal tumor that arises in children and has a grim prognosis. Nevertheless, the natural progression of the disease can be affected with a combination of resection, multiagent chemotherapy, and craniospinal radiation therapy. Finally, we have shown that survival is prolonged as children mature and when they receive radiation therapy, although these factors could not be assessed separately. 


\section{References}

1. Bambakidis NC, Robinson S, Cohen M, et al: Atypical teratoid/rhabdoid tumors of the central nervous system: clinical, radiographic and pathologic features. Pediatr Neurosurg 37: 64-70, 2002

2. Behring B, Bruck W, Goebel HH, et al: Immunohistochemistry of primary central nervous system malignant rhabdoid tumors: report of five cases and review of the literature. Acta Neuropathol 91:578-586, 1996

3. Burger PC, Yu IT, Tihan T, et al: Atypical teratoid/rhabdoid tumor of the central nervous system: a highly malignant tumor of infancy and childhood frequently mistaken for medulloblastoma: a Pediatric Oncology Group study. Am J Surg Pathol 22: 1083-1092, 1998

4. Gandhi CD, Krieger MD, McComb JG: Atypical teratoid/rhabdoid tumor: an unusual presentation. Neuroradiology 46: 834-837, 2004

5. Hilden JM, Meerbaum S, Burger P, et al: Central nervous system atypical teratoid/rhabdoid tumor: results of therapy in children enrolled in a registry. J Clin Oncol 22:2877-2884, 2004

6. Hilden JM, Watterson J, Longee DC, et al: Central nervous system atypical teratoid tumor/rhabdoid tumor: response to intensive therapy and review of the literature. J Neurooncol 40: 265-275, 1998

7. Ho DM, Hsu CY, Wong TT, et al: Atypical teratoid/rhabdoid tumor of the central nervous system: a comparative study with primitive neuroectodermal tumor/medulloblastoma. Acta Neuropathol 99:482-488, 2000

8. Olson TA, Bayar E, Kosnik E, et al: Successful treatment of disseminated central nervous system malignant rhabdoid tumor. J Pediatr Hematol Oncol 17:71-75, 1995

9. Parham DM, Weeks DA, Beckwith JB: The clinicopathologic spectrum of putative extrarenal rhabdoid tumors. An analysis of 42 cases studied with immunohistochemistry or electron microscopy. Am J Surg Pathol 18:1010-1029, 1994

10. Ronghe MD, Moss TH, Lowis SP: Treatment of CNS malignant rhabdoid tumors. Pediatr Blood Cancer 42:254-260, 2004

11. Rorke LB, Packer RJ, Biegel JA: Central nervous system atypical teratoid/rhabdoid tumors of infancy and childhood: definition of an entity. J Neurosurg 85:56-65, 1996

12. Weiss E, Behring B, Behnke J, et al: Treatment of primary malignant rhabdoid tumor of the brain: report of three cases and review of the literature. Int J Radiat Oncol Biol Phys 41: 1013-1019, 1998

Manuscript received April 19, 2005.

Accepted in final form May 18, 2005.

Address reprint requests to: Mark Krieger, M.D., 1300 North Vermont Avenue \#1006, Los Angeles, California 90027. email: MKrieger@chla.usc.edu. 